\title{
Au cour de la cardiomyopathie diabétique
}

$>$ Le diabète de type 2 (DT2) est un facteur de risque indépendant de l'insuffisance cardiaque. Ces anomalies sont cependant associées à une lipotoxicité et à une glucotoxicité cardiaques. Pourtant, les mécanismes exacts de ces toxicités cardiaques demeurent inconnus. Récemment, le phénotype cardiaque d'un modèle unique de DT2, les souris lipodystrophiques invalidées pour le gène codant la seipine (SKO), a révélé l'importance de la glucotoxicité dans le développement des anomalies cardiaques. En effet, les souris SKO présentent une cardiomyopathie associée à une surcharge en glucose, qui est corrigée par l'utilisation d'un agent hypoglycémiant, un inhibiteur du co-transporteur sodium-glucose de type 2 (SGLT2). Dans ce modèle, la glucotoxicité peut ainsi, à elle seule, générer les dysfonctions cardiaques associées au diabète. <

\section{La cardiomyopathie du sujet diabétique}

En 2013, 4,7\% de la population française, soit plus de 3 millions de personnes, étaient diabétiques [1]. Le diabète de type 2 (DT2) est une maladie qui altère la glycorégulation, conduisant à une hyperglycémie chronique, définie par une glycémie à jeun supérieure à $1,26 \mathrm{~g} / \mathrm{l}$ à deux reprises, une glycémie supérieure à 2,0 g/l accompagnée de symptômes du diabète (polyurie, polydipsie, etc.) ou une glycémie supérieure à 2,0 $\mathrm{g} / \mathrm{l}$ deux heures après une charge orale de $75 \mathrm{~g}$ de sucre [2]. II s'agit d'un trouble de l'assimilation, du stockage et de l'utilisation du glucose qui évolue en trois étapes:

- l'insulinorésistance, qui correspond à la diminution de la réponse cellulaire et tissulaire à l'action de l'insuline, - l'hyperinsulinisme, une augmentation de la sécrétion d'insuline par le pancréas en réaction à l'insulinorésistance,

- I'insulinopénie, c'est-à-dire l'épuisement progressif du pancréas, qui conduit à une diminution de la sécrétion d'insuline.

\section{Les souris}

\section{lipodystrophiques SKO} comme modèle d'étude de la glucotoxicité

Alexandre Lugat ${ }^{1}$, Michael Joubert ${ }^{2,3}$, Bertrand Cariou ${ }^{1}$, Xavier Prieur ${ }^{4}$

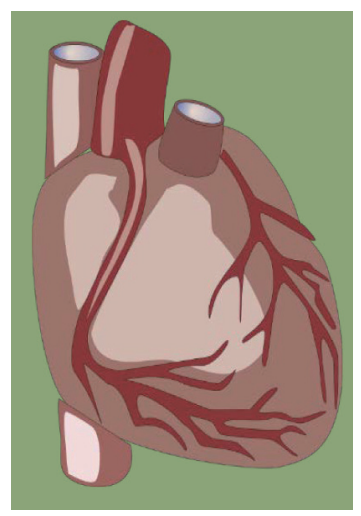

${ }^{1}$ Institut du thorax, Inserm, CNRS, univ Nantes, CHU Nantes, 8, quai Moncousu, Nantes, F-44000, France.

${ }^{2}$ Service Endocrinologie, $\mathrm{CHU}$ de Caen, Caen, F-14003, France. ${ }^{3} E A 4650$ - Unicaen - GIP Cyceron - Caen, Caen, F-14074, France. ${ }^{4}$ Institut du thorax, Inserm, CNRS, univ Nantes, Nantes, F-44000, France.

xavier.prieur@univ-nantes.fr

Le DT2 est une maladie d'évolution insidieuse et progressive, contrairement au diabète de type 1 (DTI) qui est d'origine autoimmune et conduit à une insulinopénie totale. L'origine du DT2 est multifactorielle, mais la génétique semble jouer un rôle prépondérant, à côté du surpoids et de la sédentarité. Pour l'Organisation mondiale de la santé (OMS), les facteurs de risque principaux sont le surpoids et l'obésité (notamment l'obésité abdominale), la sédentarité, l'antécédent de diabète chez la mère ou de diabète gestationnel, et l'âge [3]. L'augmentation de la prévalence du DT2 s'associe à une augmentation parallèle de celle de la cardiomyopathie diabétique, l'une des complications cardiovasculaires de cette maladie, principale cause de morbi-mortalité chez les patients [4].

Le phénotype clinique de la cardiomyopathie diabétique se caractérise par une hypertrophie du ventricule gauche (VG), une dysfonction diastolique de ce ventricule avec fraction d'éjection (FEVG) conservée (pouvant être présente même chez les diabétiques n'ayant pas d'hypertrophie du VG), une dysfonction systolique du ventricule gauche (moins fréquente que la dysfonction diastolique et de survenue plus tardive) [5].

Le diabète accélère également l'apparition d'une insuffisance cardiaque (IC) chez les patients ayant présenté un infarctus du myocarde, une hypertension artérielle ou une fibrillation atriale. La prévalence de I'IC chez ces patients est de $12 \%$ contre 1 à $4 \%$ dans la population générale [6]. 


\section{Physiopathologie de la cardiomyopathie diabétique}

Différents travaux ont permis de mettre en évidence une association entre cardiomyopathie diabétique et altération majeure du métabolisme énergétique cardiaque. Physiologiquement, le cœur présente une grande flexibilité métabolique, c'est-à-dire une capacité à utiliser les substrats disponibles selon leur abondance, et cela afin de préserver sa fonction contractile essentielle. En condition de jeûne, 60 à $90 \%$ de l'énergie du cœur proviennent de la bêta-oxydation mitochondriale des acides gras $(A G)$, à l'origine de la production d'adénosine triphosphate (ATP), une molécule hautement énergétique permettant d'assurer la contraction cardiaque [7]. En état postprandial, lorsque les niveaux sanguins de glucose et d'insuline s'élèvent, un changement métabolique se produit: l'insuline induit dans le cardiomyocyte la translocation du récepteur du glucose GLUT4 ainsi que l'activation de la 6-phosphofructo-2-kinase (PFK-2), favorisant ainsi la captation du glucose et son utilisation pour la glycolyse et la production d'ATP par le cycle de Krebs [8]. L'une des caractéristiques de la cardiomyopathie diabétique réside dans la perte de cette flexibilité métabolique, avec une baisse de ses capacités à utiliser le glucose et une plus grande dépendance vis-à-vis des AG [9]. En effet, dans un contexte dans lequel les niveaux d'AG circulants sont élevés, une augmentation de leur internalisation et un dépôt ectopique de lipides au niveau myocardique sont observés chez des patients diabétiques et dans des modèles de souris obèses et diabétiques (ob/ob et $d b / d b$ ). L'accumulation de lipides au niveau myocardique survient lorsqu'il y a inadéquation entre internalisation des $A G$ par les cellules cardiaques et oxydation de ces lipides. L'accumulation d'AG favorise alors la production d'espèces lipidiques réactives (céramides et diacylglycérol) qui contribuent à altérer l'action de l'insuline par des mécanismes de lipotoxicité $[10,11]$, elle-même impliquée dans le développement de l'insulino-résistance cardiaque, du stress oxydant et de la dysfonction mitochondriale.

Le métabolisme du glucose est lui aussi fortement altéré dans la cardiomyopathie diabétique. L’hyperglycémie et l'insulino-résistance altèrent la capacité du cœur à utiliser le glucose comme source d'énergie par glycolyse. Elles sont ainsi à l'origine d'une surcharge en glucose qui contribue à la dysfonction cardiaque : c'est le phénomène de glucotoxicité [12].

À ce jour, les contributions exactes de la lipotoxicité et de la glucotoxicité dans le développement de la cardiomyopathie diabétique demeurent inconnues. La plupart des modèles étudiés présentent en effet à la fois une accumulation ectopique de lipides au niveau cardiaque et une hyperglycémie chronique (animaux diabétiques et obèses). Le développement de nouveaux modèles animaux permettant de mieux définir les voies impliquées dans la physiopathologie de la cardiomyopathie du diabétique est donc essentiel.

\section{Le rôle central de la glucotoxicité cardiaque}

Plusieurs éléments, qui laissent supposer que la glucotoxicité joue un rôle majeur dans le développement des anomalies cardiaques asso- ciées au diabète, semblent exister. Une étude clinique récente montre ainsi que, chez les patients diabétiques atteints d'insuffisance cardiaque, ceux dont le contrôle glycémique est meilleur présentent un taux de mortalité plus faible [13].

En utilisant des biopsies cardiaques de patients atteints de DT2 et/ou obèses, il a pu être montré que les anomalies fonctionnelles des fibres cardiaques étaient plus corrélées avec le taux d'HbAlc (hémoglobine glyquée, reflétant l'historique proche de la glycémie) qu'avec l'indice d'insulino-résistance (HOMA-IR pour homeostasis model assessment of insuline resistance), ou l'indice de masse corporelle (IMC), suggérant que l'hyperglycémie est un élément clé dans le développement de la cardiomyopathie [14].

Le glucose peut altérer directement les propriétés des cardiomyocytes, notamment en diminuant la formation de myofibrilles, en particulier ceux de la chaîne lourde de myosine et de l'isoforme alpha de l'actine cardiaque [15]. De plus, des concentrations élevées de glucose sont à l'origine d'un stress cellulaire, par l'activation de la voie de signalisation mTOR (mammalian target of rapamycin) [16] pouvant conduire à une augmentation de l'apoptose des cellules cardiaques [17].

Trois voies majeures impliquées dans les effets toxiques du glucose au niveau cardiaque existent (Figure I). En effet, la surcharge en glucose facilite (1) les phénomènes de glycation ${ }^{1}$, altérant le fonctionnement des protéines cardiaques participant à la signalisation calcique, et donc à la relaxation myocardique [18, 19] ; (2) la formation de radicaux libres de l'oxygène, contribuant au stress oxydatif : par la voie des pentoses phosphates, le glucose-6-P est à l'origine de la production de substrats de la NADPH (reduced nicotinamide adenine dinucleotide phosphate) oxydase, un complexe enzymatique multiprotéique et cytosolique, capable de produire des espèces réactives de l'oxygène [21] ; (3) au niveau cardiaque, cette surcharge entraîne une activation chronique de la voie des hexosamines. Cette voie permet, à partir du glucose, de synthétiser des molécules d'UDP-GIcNAc (uridine diphosphate-N-acétylglucosamine) qui seront liées aux protéines de manière covalente. La 0-GlcNAcylation est une modification post-traductionnelle physiologique. Elle est impliquée dans différentes voies de signalisation et modifie l'activité des protéines ciblées. Au contraire de la glycation, il s'agit d'une réaction enzymatique conduisant

\footnotetext{
${ }^{1}$ La glycation est un phénomène non enzymatique de modification post-traductionnelle des protéines correspondant à la liaison covalente d'un sucre (glucose ou fructose) sur un résidu lysine ou arginine. Elle dépend de la concentration sanguine en sucre mais aussi de la durée de vie de la protéine (conditionnant la durée d'exposition au sucre) [20].
} 


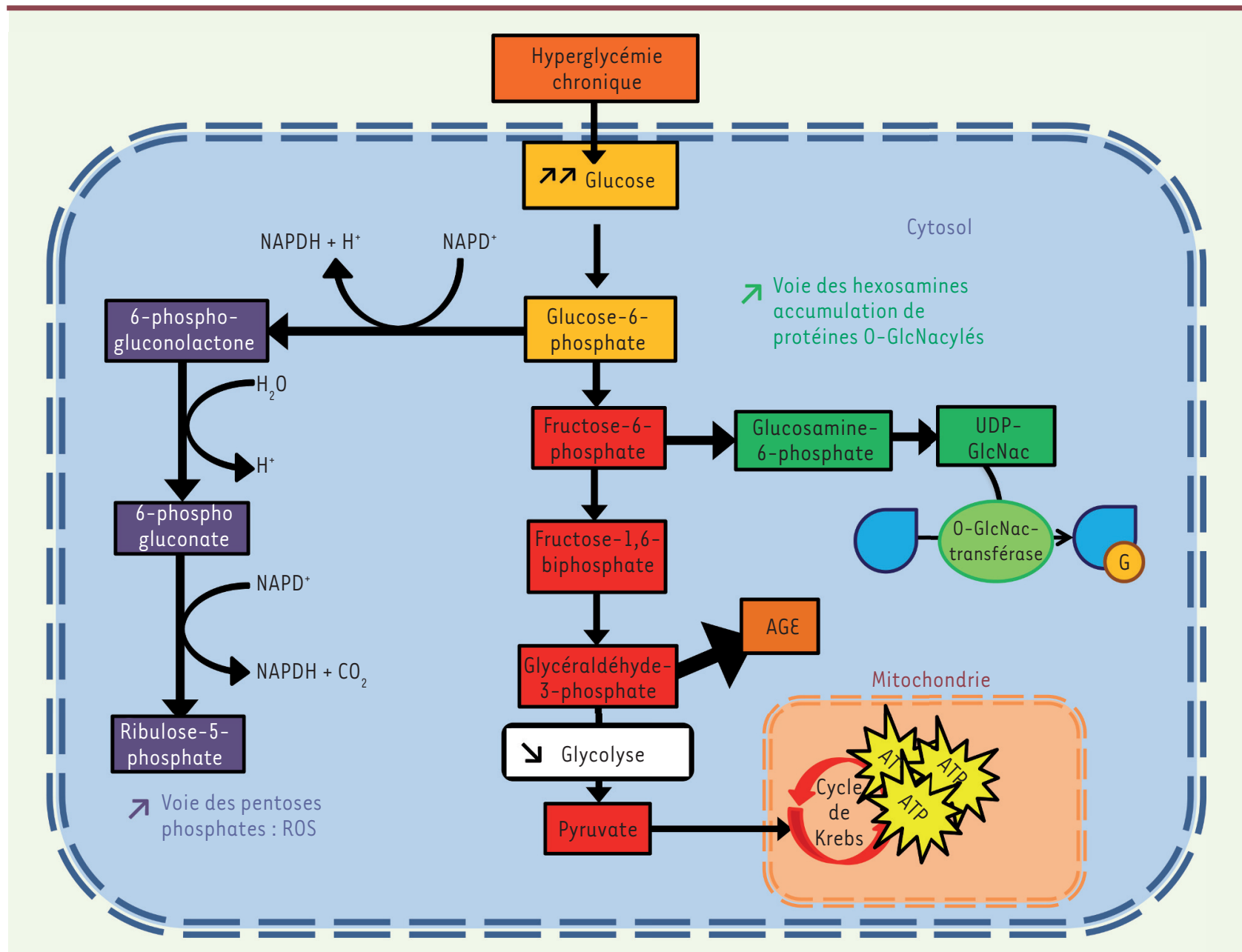

Figure 1. Mécanismes de la glucotoxicité au niveau cellulaire cardiaque. Au cours du diabète, il y a une diminution de l'internalisation du glucose par la cellule, mais aussi une diminution de la glycolyse. II en résulte une activation chronique de la voie des hexosamines avec une augmentation de la 0-GIcNAcylation des protéines pouvant altérer la signalisation calcique et la contractibilité cardiaque et une activation de la voie des pentoses phosphates créant des radicaux libres. ROS : espèces réactives de l'oxygène; AGE : produits avancés de glycation ; ATP : adénosine triphosphate; $\mathrm{NAPD}^{+}$: nicotinamide adénine dinucléotide phosphate forme oxydée ; NAPDH : nicotinamide adénine dinucléotide phosphate forme réduite; (G) : groupement $\mathrm{N}$-acétylglucosamine.

à l'addition d'une molécule de $\mathrm{N}$-acétylglucosamine (GlcNac) sur le groupement hydroxyle d'une sérine ou d'une thréonine d'une protéine [22]. L'activation chronique de cette voie a été impliquée dans le développement des anomalies cardiaques associées au diabète. La 0-GIcNAcylation participe en effet à l'altération de la contractilité cardiaque [23], de la signalisation calcique [19] ainsi qu'à la diminution de la consommation en oxygène [24].

\section{La souris lipodystrophique SKO : un modèle original pour l'exploration de la glucotoxicité cardiaque}

Les modèles animaux d'étude de la cardiomyopathie diabétique, reposent sur l'utilisation d'animaux obèses et diabétiques. Le modèle le plus classiquement décrit pour le DT2, est celui du rat Zucker fatty rat (ZFR) qui porte une mutation au niveau du récepteur de la leptine, une hormone impliquée dans la satiété. Le rat ZFR mâle développe une obésité qui est associée à une insulinorésistance caractérisée par un hyperinsulinisme, une intolérance au glucose ainsi qu'une hyperlipidémie. Il est donc impossible, avec ce modèle, de distinguer les effets respectifs de l'insulino-résistance, de la glucotoxicité, ou de la lipotoxicité.

Chez l'homme, les phénotypes de la cardiomyopathie diabétique et de ceux observés dans la lipodystrophie congénitale de Berardinelli-Seip (BSCL) sont liés à des mutations du gène $B S C L 2$ qui code la seipine, une protéine impliquée dans la genèse des goutelettes lipidiques. Nous avons donc examiné le phénotype 
cardiaque d'un modèle murin unique de diabète lipodystrophique, les souris SKO, dont le gène Bscl2 a été invalidé [25].

La lipodystrophie congénitale de Berardinelli-Seip est un syndrome autosomique récessif qui représente la forme la plus sévère de lipodystrophie généralisée avec une absence quasi complète de tissus adipeux dès la naissance, associée à une insulino-résistance puis, rapidement, à un diabète lipoatrophique. Les patients atteints de ce syndrome présentent une hypertrophie musculaire, une organomégalie avec, notamment, une hypertrophie cardiaque associée à une vitesse de croissance augmentée. Deux formes principales du syndrome ont été identifiées: BSCL1 et BSCL2. L'origine génétique de BSCL2 a été identifiée en 2001 par Magré et al. [26] au niveau du locus 1lql3 (BSCL2) qui code la seipine, dont la fonction reste inconnue, bien que des arguments plaident pour son rôle dans l'adipogenèse et la formation de gouttelettes lipidiques $[27,28]$. La cause génétique de BSCLl a, quant à elle, été identifiée plus tardivement. Elle est liée à des mutations du gène AGPAT2 (1-acylglycerol-3-phosphate 0-acyltransferase 2) codant une enzyme essentielle à la synthèse des triglycérides [29].

Le phénotype cardiaque de 44 patients présentant une lipodystrophie congénitale (dont 10 BSCL2) a été examiné [30]. Une hypertrophie du VG de sévérité variable associée à des anomalies électrocardiographiques ( $દ C G$ ) comme des ondes T négatives ou un intervalle QT allongé a ainsi été observée. Dans cette étude, et comme précédemment décrit [31], les patients BSCL2 présentaient une prévalence de cardiomyopathie plus importante. Ces anomalies électrocardiographiques et échographiques décrites chez ces patients lipodystrophiques étaient identiques à celles retrouvées dans le cadre de la cardiomyopathie associée au DT2.

Trois équipes indépendantes, dont la nôtre, ont étudié le phénotype de souris déficientes en seipine (SKO) [32-34]. Les souris SKO sont sévèrement lipodystrophiques, insulino-résistantes et diabétiques. Elles présentent une inflexibilité métabolique avec notamment une intolérance au jeûne. Les niveaux circulants d'adiponectine, une adipokine insulino-sensibilisatrice, et de leptine, une adipokine clé dans le contrôle de la satiété $[45,46](\rightarrow)$ sont particulièrement bas chez les souris SKO.

$\rightarrow$ Voir les Synthèses de S. Nicolas et al., et $\mathrm{G}$. Marcelin et K. Clément, $m / s n^{\circ} 5$, mai 2018, pages 417 et 424

Nous avons étudié le phénotype cardiaque des souris SKO et nous avons pu mettre en évidence un allongement de l'intervalle QT à l'ECG et une dysfonction diastolique en échographie (i.e une altération de la fonction de remplissage). La signalisation calcique, impliquée dans la relaxation cardiaque, est également altérée chez ces souris avec, notamment, un niveau fortement réduit de phosphorylation de phospholamban, un régulateur de la pompe calcique SERCA2 (sarcoplasmic/endoplasmic reticulum calcium ATPase 2). L'imagerie par résonnance magnétique (IRM) montre un épaississement de la paroi du VG en fin de diastole, et une baisse légère mais significative de la fraction d'éjection. L'ensemble de ces caractéristiques (dysfonction diastolique, voire systolique, et hypertrophie ventriculaire gauche) est retrouvé dans la cardiomyopathie diabétique. Sur le plan fonctionnel, les capacités physiques des souris, évaluées sur tapis roulant, se sont révélées réduites, avec une distance de course significativement dimi- nuée par rapport aux souris contrôles. Une corrélation positive entre glycémie et deux paramètres cardiaques majeurs (épaisseur de la paroi du VG et fraction d'éjection) a, par la suite, également été mise en évidence : les souris les plus hyper-glycémiques présentant l'hypertrophie ventriculaire gauche et la dysfonction cardiaque les plus marquées.

L'analyse du métabolisme énergétique cardiaque des souris SKO a été examinée en parallèle. En ce qui concerne le métabolisme lipidique, il n'a pas été possible de mettre en évidence de différence en termes de stéatose myocardique, d'internalisation des $A G$, d'accumulation de triglycérides, ni d'espèces lipidiques toxiques (céramides ou di-acyl glycérol), en comparaison avec les souris contrôles. La cardiomyopathie des souris SKO ne semble donc pas être liée à un mécanisme de lipotoxicité. Ce modèle murin ne pouvait donc être utilisé comme modèle de stéatose cardiaque.

Concernant le métabolisme glucidique cardiaque des souris, une insulinorésistance cardiaque, accompagnée d'une captation accrue de glucose par le cœur, mesurée par tomodensitométrie d'émission de positons au 18-fluorodésoxyglucose (T\&P- $\left.{ }^{18} \mathrm{FDG}\right)$, a été observée. La consommation en oxygène, évaluée par ${ }^{11} C$-acétate TEP, est également diminuée, témoignant d'une dysfonction mitochondriale. La glucotoxicité apparaît donc jouer un rôle clé dans le développement de la cardiomyopathie des souris SKO. Les voies de signalisation impliquées dans ce mécanisme de glucotoxicité cardiaque ont donc été explorées (Figure 2A).

\section{La 0-GlcNAcylation}

La glucotoxicité repose sur la formation de produits de glycation, sur un stress oxydant et/ou une activation chronique de la voie des hexosamines. Chez les souris SKO, l'accumulation de produits de glycation, ou de stress oxydant, n'est pas observée. En revanche, la voie des hexosamines est activée chroniquement. Les souris SKO présentent une accumulation au niveau cardiaque de protéines 0-GIcNAcylées, avec en particulier un accroissement de I'O-GIcNAcylation du facteur de transcription Fox0l (Figure 2A). L'O-GlcNAcylation de Fox01 augmente son activité. Au contraire, son inhibition empêche le développement de la cardiomyopathie métabolique. L'hyperactivité de FoxOl conduit en effet au changement métabolique qui réduit la captation du glucose par le cœur au profit des lipides. Fox01 active la pyruvate déshydrogénase kinase 4 (PDK4), dont le rôle est de diminuer l'oxydation du glucose en phosphorylant le complexe pyruvate déshydrogénase, empêchant ainsi le glucose d'entrer dans la mitochondrie 


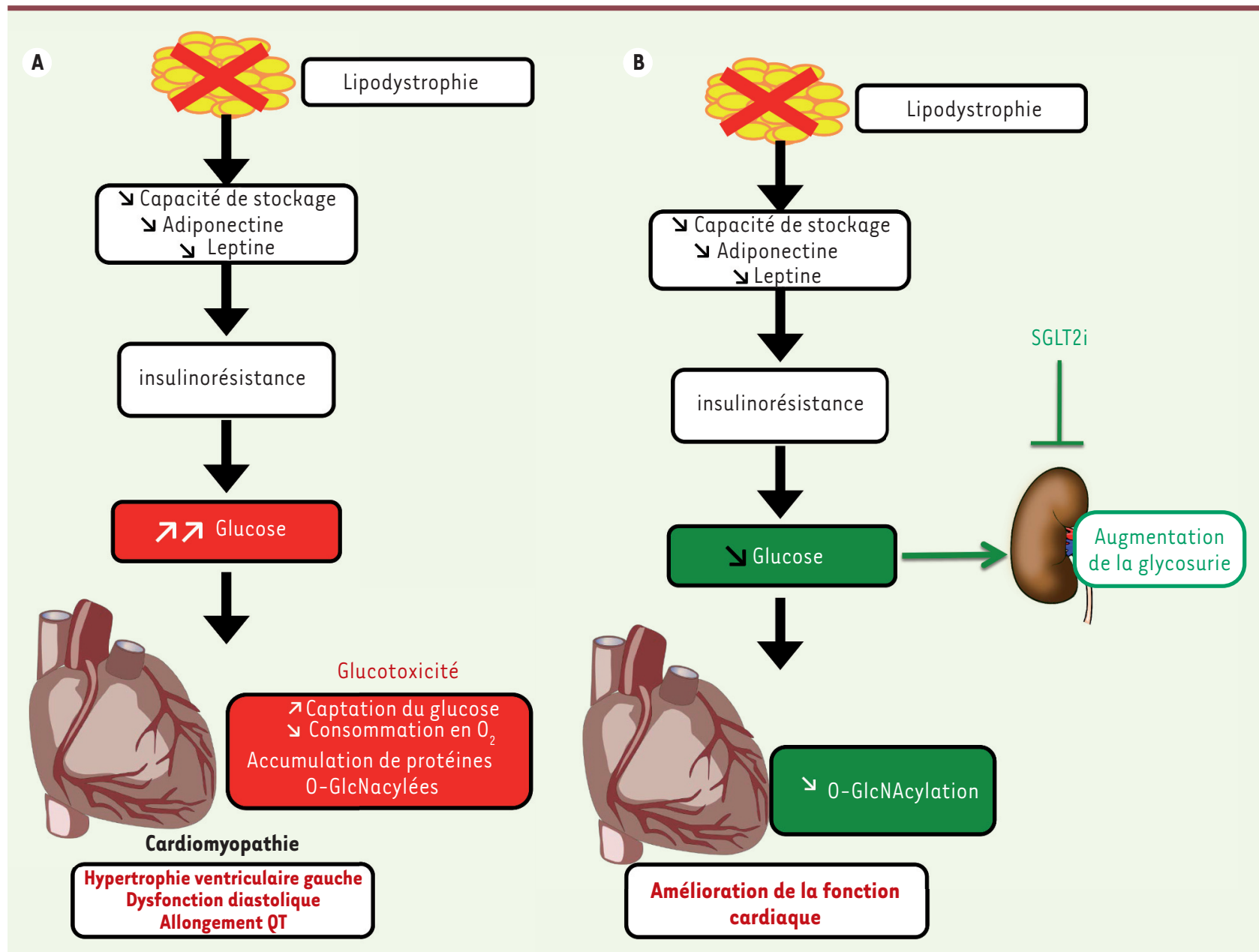

Figure 2. Les souris SKO présentent une cardiomyopathie en partie corrigée par les SGLT2i. A. Les souris lipodystrophiques présentent une diminution des taux circulant d'adiponectine et de leptine ainsi qu'une stéatose hépatique et une insulinorésistance entrainant un diabète. Au niveau cardiaque : dysfonction diastolique, hypertrophie ventriculaire gauche, augmentation de la captation du glucose et diminution de la consommation en oxygène, augmentation de l'intervalle PT à l'électrocardiogramme, augmentation du taux de protéines 0 -glcNAcylées. B. Le traitement par la dapagliflozine entraîne une normalisation de la glycémie par augmentation de la glycosurie. Ceci conduit à une diminution de l'hypertrophie ventriculaire gauche et une amélioration de la fonction cardiaque. Les niveaux de protéines 0 -glcNAcylées sont abaissés.

[35]. L'inactivation cardiaque de Fox01 dans des modèles animaux a démontré son effet cardioprotecteur [36]. Nous avons donc émis l'hypothèse que l'hyperglycémie chronique chez les souris SKO entraînerait au niveau cardiaque une activation de la voie des hexosamines, ce qui affecterait le fonctionnement cardiaque en diminuant la flexibilité métabolique cardiaque, mais aussi, altérerait la contractilité et la signalisation calcique par l'accumulation de protéines 0-GIcNAcylées.

\section{Les inhibiteurs de SGLT2 : un traitement prometteur de la cardiomyopathie diabétique?}

Afin d'étudier la réversibilité de l'atteinte cardiaque et de confirmer I'hypothèse de la glucotoxicité cardiaque, les souris SKO ont été traitées par le dapagliflozine, un inhibiteur de SGLT2 (sodium-glucose co-transporter-2) (SGLT2i) qui diminue l'hyperglycémie en augmentant la glycosurie. SGLT2 est un cotransporteur membranaire sodium/glucose permettant la réabsorption du glucose dans le tubule rénal proximal. Son inhibition diminue ainsi la glycémie et la concentration d'HbAlc en augmentant la glycosurie, et abaisse la pression artérielle par une diurèse osmotique. Elle diminue le poids corporel par fuite calorique au niveau urinaire. Les souris SKO traitées par le dapagliflozine présentent une amélioration de la fonction diastolique, de la fraction d'éjection ventriculaire gauche, ainsi qu'une diminution de l'hypertrophie myocardique. Les niveaux de phosphorylation de phospholamban sont également normalisés, ce qui est cohérent avec une 


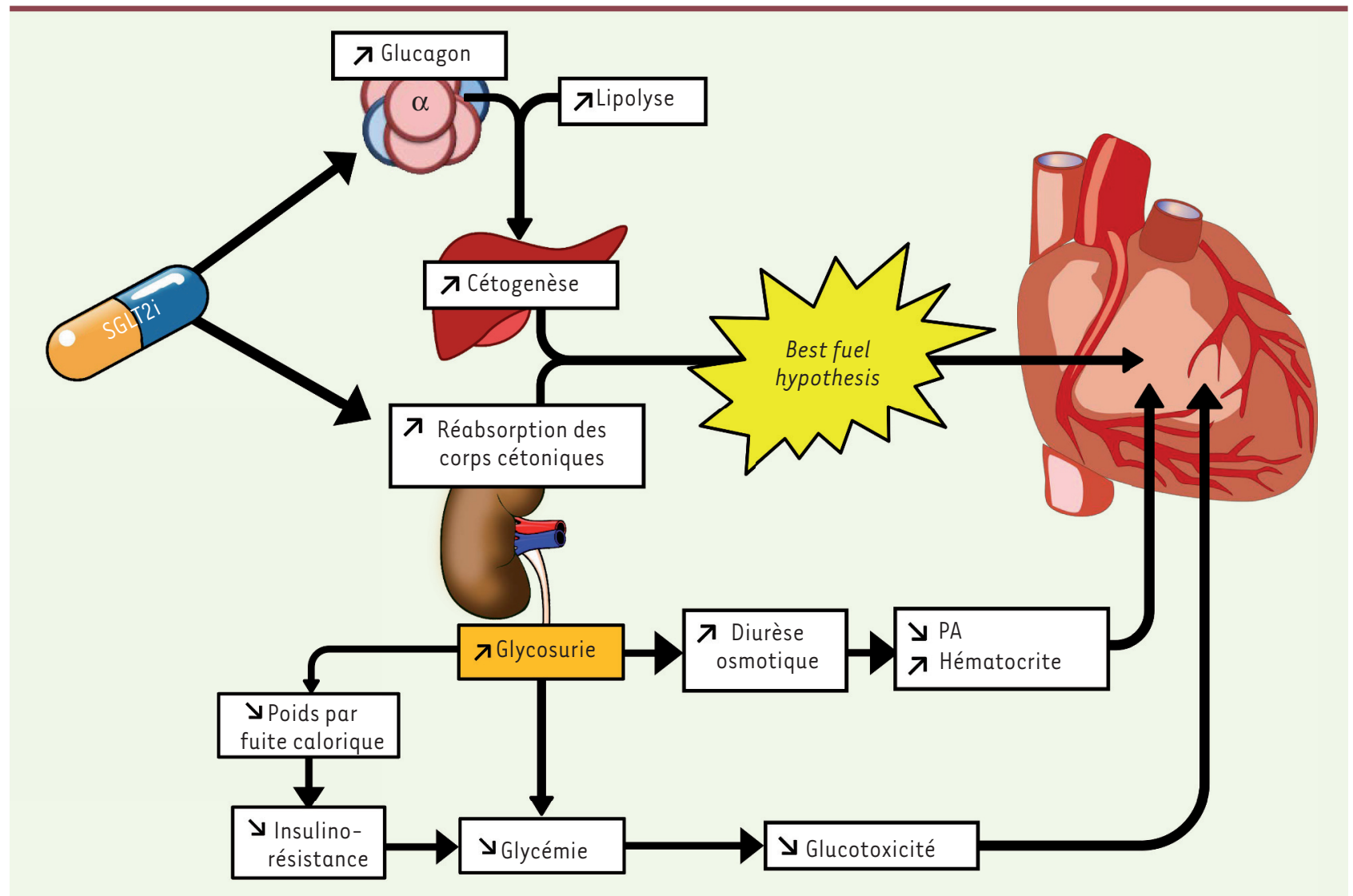

Figure 3. Mécanismes cardioprotecteurs potentiels des SGLT2i. Diminution de la glycémie indépendante de l'insuline par augmentation de la glycosurie, diminution de la pression artérielle (PA) et augmentation de l'hématocrite. Perte de poids et diminution de l'insulinorésistance. Augmentation de la cétogenèse : augmentation de la lipolyse, action directe sur les cellules alpha pancréatiques augmentant la glucagonémie et augmentation de la réabsorption rénale des corps cétoniques. SGLT2i : inhibiteur du cotransporteur sodium/glucose de type 2 ; Best fuel hypothesis : hypothèse du meilleur carburant.

meilleure capacité de relaxation du myocarde. En parallèle, une diminution du niveau général des protéines 0 -GIcNAcylées, en particulier de la fraction 0-GlcNAcylée de Fox01, est observée.

L'ensemble de ces résultats montre que, dans le modèle de souris SKO, la glucotoxicité peut, en l'absence de lipotoxicité, engendrer une dysfonction cardiaque chez ces animaux. Nous n'avons pas observé d'accumulation d'espèces réactives de l'oxygène, ni de produits avancés de glycation (AGE). L'activation de la voie des hexosamines semble donc jouer un rôle central dans le développement de la cardiomyopathie des souris SKO. La dapagliflozine exercerait ainsi son effet cardioprotecteur en diminuant la surcharge en glucose et en empêchant l'accumulation de protéines 0-GlcNAcylées (Figure 2B).

Cette protection cardiaque des inhibiteurs de SGLT2 a été démontrée chez l'homme, dans l'étude multicentrique EMPA-REG qui a évalué la sécurité d'un autre inhibiteur de SGLT2 (I'empagliflozine) avec une diminution de $38 \%$ de la mortalité cardiovasculaire, et de $32 \%$ des hospitalisations pour insuffisance cardiaque chez les patients atteints de DT2 [37]. L'étude CANVAS, utilisant la canagliflozine, a montré des résultats similaires, suggérant un effet de classe pour les inhibiteurs de type SGLT2i [38].
De nombreux travaux tentent d'élucider les mécanismes par lesquels les SGLT2i présentent des effets bénéfiques. Les effets des SGLT2i, qui sont uniques comparativement aux autres antidiabétiques, suggèrent que la baisse de la glycémie ne peut, à elle seule, expliquer le bénéfice cardiovasculaire que l'on observe. Contrairement aux autres antidiabétiques, les SGLT2i entraînent une diminution de la concentration de glucose plasmatique sans, théoriquement, favoriser son internalisation par les cellules puisque ces traitements n'ont d'action ni sur la sensibilité à l'insuline, ni sur l'insulinosécrétion. II est alors possible de spéculer que ce mécanisme d'action particulier puisse limiter l'afflux de glucose intracellulaire et donc la glucotoxicité [39].

L'une des hypothèses expliquant ce processus est dite «fuel hypothesis» (ou «super carburant») : sous traitement, la baisse non insulino-dépendante de la glycémie entraînerait une augmentation des niveaux circulants de glucagon, mimant des conditions de jeûne et favorisant la production de corps cétoniques, dont le 
bêta-hydroxybutyrate, jusqu'à l'acidocétose euglycémique [40, 41$]$. Les mécanismes de cette cétogenèse induite par les SGLT2i demeurent discutés, mais ils impliqueraient (Figure 3) : (1) une baisse de la glycémie, indépendamment de l'insuline, entraînant une diminution de l'insuline endogène pouvant conduire à l'augmentation de la lipolyse et à une production hépatique de corps cétoniques; (2) une augmentation de la production de glucagon via un rôle direct sur le transporteur SGLT2 qui serait exprimé au niveau de la cellule alpha des îlots de Langerhans. Les taux de glucagon supraphysiologiques, sans insuline pour contrebalancer son action, augmentant la lipolyse et donc la cétogenèse par action directe sur le tissu adipeux [42] ; (3) une augmentation de la réabsorption rénale de corps cétoniques.

Le bêta-hydroxybutyrate pourrait constituer un «super carburant » facilement utilisable par un cœur qui présente des défauts d'utilisation de l'énergie et une dysfonction mitochondriale. L'oxydation mitochondriale du bêta-hydroxybutyrate est en effet plus efficace, augmentant l'activité cardiaque et réduisant la consommation d'oxygène [43]. L'action des SGLT2i sur les reins diminue la pression artérielle et augmente l'hématocrite, ce qui pourrait participer à l'effet bénéfique que l'on observe [44].

\section{Conclusion}

Les mécanismes moléculaires menant à la cardiomyopathie diabétique sont multiples et encore débattus. Deux mécanismes principaux sont à prendre en compte : la glucotoxicité et la lipotoxicité. Cependant, leur implication respective reste difficile à individualiser dans cette pathologie qui implique diabète/hyperglycémie et obésité/ hyperlipidémie. L'étude d'une pathologie humaine rare, la lipodystrophie congénitale généralisée de Berardinelli-Seip, par l'utilisation d'un modèle murin original, a permis de révéler les effets de la glucotoxicité qui conduit au développement de la cardiomyopathie diabétique. En l'absence de lipotoxicité, la glucotoxicité peut, à elle seule, générer des dysfonctions métaboliques et une cardiomyopathie chez les souris SKO. Nous n'avons observé ni stress oxydatif ni accumulation de produits avancés de la glycation, et l'activation chronique de la voie des hexosamines semble jouer un rôle central dans le développement de la cardiomyopathie des souris SKO. L'amélioration des paramètres cardiaques par les SGLT2i est associée à une normalisation de la voie des hexosamines.

Ce modèle unique de souris non obèses diabétiques permet l'exploration de la glucotoxicité de manière isolée afin de mieux comprendre son rôle dans le développement de la cardiopathie diabétique, ainsi que les effets cardioprotecteurs des SGLT2i, premier antidiabétique à démontrer une diminution des évènements cardiovasculaires majeurs et des hospitalisations pour insuffisance cardiaque. $\diamond$

\section{SUMMARY}

At the heart of diabetic cardiomyopathy: Bscl2 knockout mice to investigate glucotoxicity

Type 2 diabetes mellitus (T2DM) is a well-recognized independent risk factor for heart failure (HF). T2DM is associated with altered cardiac energy metabolism, leading to ectopic lipid accumulation and glucose overload. However, the relative contribution of these two parameters remains unclear. In order to get new insight into the mechanism involved in diabetic cardiomyopathy, the cardiac phenotype of a unique T2DM mice model has been performed: the seipin knockout mice (SKO). Cardiac phenotyping revealed a diastolic dysfunction associated with hyperglycemia in these mice with a chronic activation of the hexosamine biosynthetic pathway (HBP), suggesting a glucose overload. An inhibitor of the renal sodium/ glucose cotransporter 2 (SGLT2), dapagliflozin, successfully prevented the development of cardiomyopathy in SKO mice. This is particularly relevant, given that SGLT2i treatment reduces cardiovascular event in T2DM patients. Therefore, glucose lowering appears an important therapeutic target to prevent cardiac dysfunction associated with T2DM. $\diamond$

\section{LIENS D'INTÉRÊT}

Michael Joubert a conduit des essais cliniques pour Lilly, Novonordisk, Sanofi, Takeda, Bristol Myers Squibb, Novartis, Astrazeneca, Boehringer Ingelheim, Janssen-Cilag. Bertrand Cariou est consultant pour AstraZeneca, Lilly, Sanofi et Takeda. Michael Joubert, Xavier Prieur et Bertrand Cariou ont un contrat de collaboration avec Boehringer Ingelheim sur l'étude des effets cardiaques de l'Empagliflozine.

\section{RÉFÉRENCES}

1. Fosse--Edorh S, Mandereau-Bruno L, Regnault N. Le poids des complications liées au diabète en France en 2013. Synthèse et perspectives. Bull Epidemiol Hebd 2015 ; 34-35: 619-25.

2. Haute Autorité de Santé. Prévention et dépistage du diabète de type 2 et des maladies liées au diabète. Paris: HAS, 2014.

3. Organisation Mondiale de la Santé. Rapport mondial sur le diabète. Genève : OMS, 2016.

4. Danaei G, Finucane MM, Lu Y, Singh GM, et al. National, regional, and global trends in fasting plasma glucose and diabetes prevalence since 1980: systematic analysis of health examination surveys and epidemiological studies with 370 country-years and 2.7 million participants. Lancet Lond Engl $2011 ; 378$ : 31-40.

5. Aneja A, Tang WHW, Bansilal S, et al. Diabetic cardiomyopathy: insights into pathogenesis, diagnostic challenges, and therapeutic options. Am J Med $2008 ; 121: 748-57$.

6. MacDonald MR, Jhund PS, Petrie MC, et al. Discordant short- and longterm outcomes associated with diabetes in patients with heart failure: importance of age and sex. Clinical Perspective: A population study of 5.1 million people in Scotland. Circ Heart Fail 2008 ; $1: 234-41$.

7. Stanley WC, Recchia FA, Lopaschuk GD. Myocardial substrate metabolism in the normal and failing heart. Physiol Rev 2005 ; 85 : 1093-129.

8. Bertrand L, Horman S, Beauloye $C$, et al. Insulin signalling in the heart. Cardiovasc Res $2008 ; 79: 238-48$.

9. Young ME, McNulty P, Taegtmeyer H. Adaptation and maladaptation of the heart in diabetes. Part II: potential mechanisms. Circulation 2002 ; 105 : 1861-70.

10. Abel ED, O'Shea KM, Ramasamy R. Insulin resistance: metabolic mechanisms and consequences in the heart. Arterioscler Thromb Vasc Biol 2012 ; 32 : 2068-76.

11. Zlobine I, Gopal K, Ussher JR. Lipotoxicity in obesity and diabetes-related cardiac dysfunction. Biochim Biophys Acta 2016 ; 1861 : 1555-68.

12. Kolwicz SC, Tian R. Glucose metabolism and cardiac hypertrophy. Cardiovasc Res $2011 ; 90: 194-201$.

13. Elder DHJ, Singh JSS, Levin D, et al. Mean HbAlc and mortality in diabetic individuals with heart failure: a population cohort study. Eur J Heart Fail 2016 ; $18: 94-102$. 


\section{RÉFÉRENCES}

14. Montaigne D, Marechal X, Coisne A, et al. Myocardial contractile dysfunction is associated with impaired mitochondrial function and dynamics in type 2 diabetic but not in obese patients. Circulation $2014 ; 130: 554-64$.

15. Dyntar D, Sergeev P, Klisic J, et al. High glucose alters cardiomyocyte contacts and inhibits myofibrillar formation. J Clin Endocrinol Metab 2006 ; 91 : 1961-7.

16. Sen $S$, Kundu BK, Wu HC, et al. Glucose regulation of load-induced mTOR signaling and $\varepsilon R$ stress in mammalian heart. J Am Heart Assoc Cardiovasc Cerebrovasc Dis 2013 ; 2 : e004796.

17. Cai L, Li W, Wang G, et al. Hyperglycemia-induced apoptosis in mouse Mmyocardium: mitochondrial cytochrome c-mediated caspase-3 activation pathway. Diabetes $2002 ; 51: 1938$ 48.

18. Tian C, Alomar F, Moore CJ, et al. Reactive carbonul species and their roles in sarcoplasmic reticulum $\mathrm{Ca}^{2+}$ cycling defect in the diabetic heart. Heart Fail Rev $2014 ; 19: 101-12$.

19. Shao CH, Rozanski G), Patel KP, et al. Dyssynchronous (non-uniform) $\mathrm{Ca}^{2+}$ release in myocytes from streptozotocin-induced diabetic rats. J Mol Cell Cardiol 2007 ; 42 : 234-46.

20. Thornalley PJ, Langborg A, Minhas HS. Formation of glyoxal, methylglyoxal and 3-deoxyglucosone in the glycation of proteins by glucose. Biochem J $1999 ; 344: 109-16$.

21. Brahma MK, Pepin ME, Wende AR. My Sweetheart is broken: role of glucose in diabetic cardiomyopathy. Diabetes Metab J 2017 ; 41 : 1-9.

22. Wright JN, Collins HE, Wende AR, et al. O-GIcNAcylation and cardiovascular disease. Biochem Soc Trans $2017 ; 45: 545-53$.

23. Ramirez-Correa GA, Jin W, Wang Z, et al. 0-linked GIcNAc modification of cardiac myofilament proteins: A novel regulator of myocardial contractile function. Circ Res 2008 ; 103 : 1354-8.

24. Hu Y, Suarez J, Fricovsky $\varepsilon$, et al. Increased enzymatic 0 -GIcNAcylation of mitochondrial proteins impairs mitochondrial function in cardiac myocytes exposed to high glucose. J Biol Chem 2009; $284: 547-55$.

25. Joubert M, Jagu B, Montaigne D, et al. The sodium-glucose cotransporter 2 inhibitor dapagliflozin prevents cardiomyopathy in a diabetic lipodystrophic mouse model. Diabetes $2017 ; 66: 1030-40$.

26. Magré J, Delépine M, Khallouf $\varepsilon$, et al. Identification of the gene altered in Berardinelli-Seip congenital lipodystrophy on chromosome 1lq13. Nat Genet $2001 ; 28: 365-70$.

27. Szymanski KM, Binns D, Bartz $R$, et al. The lipodystrophy protein seipin is found at endoplasmic reticulum lipid droplet junctions and is important for droplet morphology. Proc Natl Acad Sci USA $2007 ; 104: 20890-5$.

28. Cartwright BR, Goodman JM. Seipin: from human disease to molecular mechanism. J Lipid Res $2012 ; 53: 1042-55$.

29. Agarwal AK, Arioglu $\varepsilon$, De Almeida $S$, et al. AGPAT2 is mutated in congenital generalized lipodystrophy linked to chromosome 9q34. Nat Genet 2002 ; $31: 21-3$.

30. Lupsa BC, Sachdev V, Lungu A0, et al. Cardiomyopathy in congenital and acquired generalized lipodystrophy. Medicine (Baltimore) $2010 ; 89: 245-50$.

31. Agarwal AK, Simha V, Oral $\varepsilon A$, et al. Phenotypic and genetic heterogeneity in congenital generalized lipodystrophy. J Clin Endocrinol Metab $2003 ; 88$ : 4840-7.
32. Cui $X$, Wang $Y$, Tang $Y$, et al. Seipin ablation in mice results in severe generalized lipodystrophy. Hum Mol Genet $2011 ; 20$ : 3022-30.

33. Chen $W$, Chang B, Saha P, et al. Berardinelli-Seip congenital lipodystrophy $2 /$ seipin is a cell-autonomous regulator of lipolysis essential for adipocyte differentiation. Mol Cell Biol 2012 ; 32 : 1099-111.

34. Prieur X, Dollet L, Takahashi M, et al. Thiazolidinediones partially reverse the metabolic disturbances observed in Bscl2/seipin-deficient mice. Diabetologia 2013 ; 56 : 1813-25.

35. Chistiakov DA, Orekhov AN, Bobryshev YV. The impact of FOXO-1 to cardiac pathology in diabetes mellitus and diabetes-related metabolic abnormalities. Int J Cardiol 2017 ; 245 : 236-44.

36. Ferrannini $\varepsilon$. Sodium-glucose co-transporters and their inhibition: clinical physiology. Cell Metab $2017 ; 26: 27-38$

37. Zinman B, Wanner C, Lachin JM, et al. Empagliflozin, cardiovascular outcomes, and mortality in type 2 diabetes. EMPA-REG outcome investigators. N Engl J Med 2015 ; 373 : 2117-28.

38. Neal B, Perkovic V, Mahaffey KW, et al. Canagliflozin and cardiovascular and renal events in type 2 diabetes. $N$ Engl J Med 2017 ; 377 : 644-57.

39. Staels B. Cardiovascular protection by sodium glucose cotransporter 2 inhibitors: potential mechanisms. Am J Cardiol 2017 ; 120 : S28-36.

40. Taylor SI, Blau JE, Rother KI. SGLT2 Inhibitors may predispose to ketoacidosis. J Clin Endocrinol Metab 2015 ; 100 : 2849-52.

41. Ferrannini $\varepsilon$, Mark M, Mayoux $\varepsilon$. CV Protection in the EMPA-REG OUTCOME trial: a Thrifty substrate hypothesis. Diabetes Care 2016 ; 39 : 1108-14.

42. Liljenquist JE, Bomboy JD, Lewis SB, et al. Effects of glucagon on lipolysis and ketogenesis in normal and diabetic men. J Clin Invest $1974 ; 53: 190-7$.

43. Sato K, Kashiwaya $Y$, Keon CA, et al. Insulin, ketone bodies, and mitochondrial energy transduction. FASEB J 1995 ; $9: 651-8$.

44. Scheen AJ, Delanaye P. Effects of reducing blood pressure on renal outcomes in patients with type 2 diabetes: focus on SGLT2 inhibitors and EMPA-REG OUTCOME. Diabetes Metab 2017 ; 43 : 99-109.

45. Nicolas S, Chabry J, Guyon A, et al. L'adiponectine : un anti-inflammatoire et antidépresseur endogène? Med Sci (Paris) 2018 ; 34 : 417-23.

46. Marcelin G, Clément K. La fibrose du tissu adipeux : un facteur aggravant de l'obésité. Med Sci (Paris) 2018 ; 34 : 424-31.

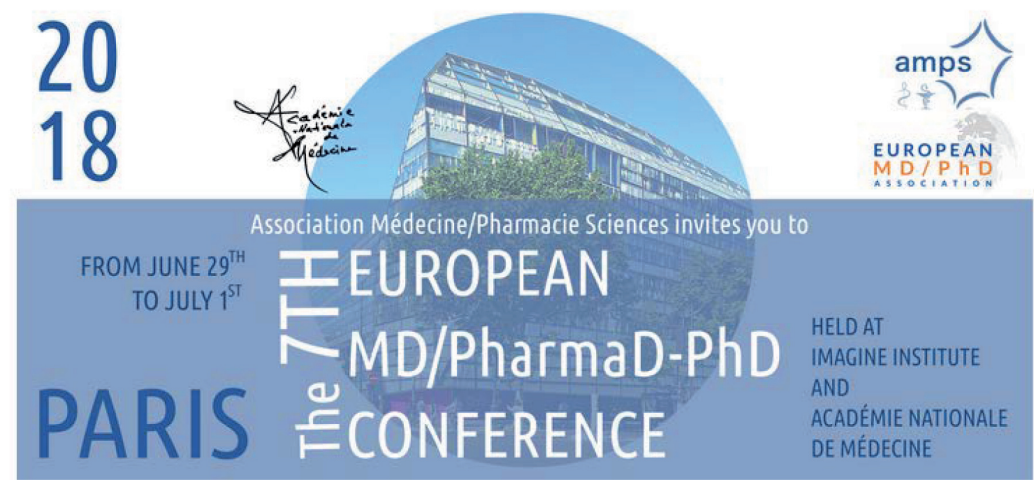

L'AMPS (Association Médecine Pharmacie Sciences), en partenariat avec l'EMPA (European MD PhD Association), organise son prochain congrès annuel du 29 Juin au 1er Juillet, à l'Académie de Médecine et à l'Institut Imagine. Au programme : de la génétique (Pr Laurent Abel), de la cancérologie (Pr Hugues de Thé, Pr Guido Kroemer), de l'ethnopharmacologie (Dr Jacques Fleurentin), et de nombreuses autres présentations. Ces trois journées seront rythmées par ces conférences, entrecoupées de workshops (avec notamment Pr Boris Barbour et Pr Laurent Mesnard), de présentations étudiantes (dont les meilleures seront primées), d'une table ronde sur l'entrepreneuriat (à laquelle participera le Pr Jean Pierre Kinet, de Harvard) et de nombreuses autres surprises. Pour plus d'informations, n'hésitez pas à consulter le site de l'AMPS : http://www.ampsasso.fr

TIRÉS À PART

$X$. Prieur 